Research article

\title{
Comparison of the MicroScan, VITEK 2, and Crystal GP with I6S rRNA sequencing and MicroSeq 500 v2.0 analysis for coagulase-negative Staphylococci
} Miyoung $\mathrm{Kim}^{\dagger 1}$, Se Ran $\mathrm{Heo}^{2}$, Soon Hee Choi ${ }^{2}$, Hyelin Kwon'2, Jeong Su Park¹, Moon-Woo Seong 3 , Do-Hoon Lee ${ }^{3}$, Kyoung Un Park*1,2,4, Junghan Song 1,2,4 and Eui-Chong Kim ${ }^{1,4}$

\author{
Address: ${ }^{1}$ Department of Laboratory Medicine, Seoul National University Hospital, 101 Daehang-no, Jongno-gu, Seoul, South Korea, ${ }^{2}$ Department \\ of Laboratory Medicine, Seoul National University Bundang Hospital, 300 Gumi-dong, Bundang-gu, Seongnam-si, Gyeonggi-do, South Korea, \\ ${ }^{3}$ Department of Laboratory Medicine, National Cancer Center, 809 Madu-1-dong, Ilsandong-gu, Goyang, Gyeonggi-do, South Korea and \\ ${ }^{4}$ Department of Laboratory Medicine, Seoul National University College of Medicine, 101 Daehang-no, Jongno-gu, Seoul, South Korea \\ Email: Miyoung Kim - rabbit790622@gmail.com; Se Ran Heo - sellyozoo@hanmail.net; Soon Hee Choi - soon2ya@snubh.org; \\ Hyelin Kwon - hyelinn@hanmail.net; Jeong Su Park - mdjs0721@naver.com; Moon-Woo Seong - mwseong@ncc.re.kr; Do- \\ Hoon Lee - dhlee@ncc.re.kr; Kyoung Un Park* - m91w95@dreamwiz.com; Junghan Song - songjhcp@snu.ac.kr; Eui- \\ Chong Kim - euichong@snu.ac.kr \\ * Corresponding author †Equal contributors
}

Published: 23 December 2008

BMC Microbiology 2008, 8:233 doi:10.1 I86/I47I-2180-8-233
Received: 10 June 2008

Accepted: 23 December 2008

This article is available from: http://www.biomedcentral.com/I47I-2180/8/233

(c) 2008 Kim et al; licensee BioMed Central Ltd.

This is an Open Access article distributed under the terms of the Creative Commons Attribution License (http://creativecommons.org/licenses/by/2.0), which permits unrestricted use, distribution, and reproduction in any medium, provided the original work is properly cited.

\begin{abstract}
Background: Three phenotypic identification systems (MicroScan, VITEK 2, and Crystal GP) were evaluated for their accuracy to identify coagulase-negative staphylococci (CNS). A total of 120 clinical isolates confirmed to be CNS via I6S rRNA sequencing and analysis with the MicroSeq 500 v2.0 database were assessed.

Results: The MicroScan, VITEK 2, and Crystal GP systems correctly identified $82.5 \%, 87.5 \%$, and $67.5 \%$ of the isolates, respectively. Misidentification was the main problem in MicroScan (10.8\%) and Crystal GP (23.3\%) systems, whereas the main problem of VITEK 2 was low-level discrimination (7.5\%).

Conclusion: None of the 3 phenotypic systems tested could accurately and reliably identify CNS at the species level. Further verifications such as biochemical testing or I6S rRNA sequencing together with analysis using a comparable database might be helpful in this regard.
\end{abstract}

\section{Background}

Because of their ubiquity and low virulence, coagulasenegative staphylococci (CNS) have generally been considered to be nonpathogens or simple contaminants. Recently, their clinical significance is being increasingly recognized with the elucidation of their pathogenicity. CNS can form biofilms and have been demonstrated to exhibit antibiotic resistance $[1,2]$. S. epidermidis, S. haemolyticus, and $S$. lugdunensis, to a lesser extent, are wellknown etiological agents of implanted device-mediated infections [2-5]. S. saprophyticus can cause communityacquired infections of the uropoietic tract. Hence, specieslevel identification of CNS is necessary for correct guidance of clinicians in terms of appropriate treatment strat- 
egy, and a wide variety of identification methods have been proposed.

Many automated phenotypic identification systems are commercially available, including the MicroScan (Dade Behring, West Sacramento, CA, USA), VITEK 2 (bioMérieux, Maray l'Etoile, France), and Crystal GP (Becton Dickinson, Sparks, MD, USA) systems. On the basis of metabolic activities and/or morphological features, these systems enable microbiologists to identify bacterial isolates at the species level with greater ease, accuracy, and rapidity than that previously achieved [6]. However, these systems have several potential problems: (i) different strains in one species may not exhibit a specific characteristic, (ii) isolates from old cultures may not show the expected biochemical patterns, (iii) isolates from a host who has undergone long-term antimicrobial therapy may alter their typical biochemical characteristics, (iv) the same strain may not yield the same results in repeated tests, (v) the databases have data on a limited number of species, (vi) phenotypic variation may affect the accuracy of species-level identification by automated phenotypic systems, and (vii) phenotypic systems often suggest 2 or more designations with comparable probability levels [69].

Recently, genotypic methods are emerging as the new standard for bacterial identification in automated laboratories [4,6,7,10-14]. 16S ribosomal RNA (rRNA) sequences comprising both variable and conserved regions allow for clear differentiation between organisms not only at the species level but also at the subspecies level [10]. These sequences permit better identification of rare or phenotypically aberrant species as well as nonculturable bacteria. The MicroSeq 500 system (Applied Biosystems Inc. [ABI], Foster City, CA, USA) is a commercially available software system for 16S rRNA analysis.

Despite the increasing clinical significance of CNS and the growing use of automated phenotypic systems in clinical laboratories, few studies have systematically evaluated the accuracy of these systems $[4,9,15]$. In the present study, the accuracy of 3 commercial phenotypic systems (MicroScan, VITEK 2, and Crystal GP) for CNS identification was compared. 16S rRNA sequencing and MicroSeq 500 analysis were used as references [10-12,16-19].

\section{Results}

\section{Clinical isolates}

By using in-house primers and the MicroSeq 500 v2.0 database, we successfully identified all the 120 clinical isolates with more than $97 \%$ matches (data not shown). The identified species were as follows: 16 S. capitis, 4 S. caprae, 3 S. cohnii, 53 S. epidermidis, 4 S. haemolyticus, 25 S. hominis, 6 S. lugdunensis, 1 S. saprophyticus, 2 S. simulans, and 6 S. warneri. Blood culture specimens primarily contained S. epidermidis, whereas a variety of uncommon CNS were isolated from pitted keratolysis specimens. The result reflected the typical distribution of staphylococcal species detected routinely in microbiology laboratories.

\section{Identification using MicroScan}

The overall performances of 3 phenotypic systems are summarized in Table 1 and the incorrect identification results are listed in Table 2. Of 120 isolates, correct identification, low-level discrimination and misidentification were $82.5 \%$ (99), $6.7 \%(8)$ and $10.8 \%$ (13), respectively. Nonidentification was not observed. Three S. caprae isolates were not correctly identified (isolate number: M211, M215, and M225), because the species $S$. caprae is not included in the MicroScan version 2.0 or 2.1 database. One of the S. epidermidis isolates (M106) was misidentified as $S$. aureus.

\section{Identification using VITEK 2}

In the VITEK 2 analysis, correct identification, low-level discrimination and misidentification were $87.5 \%$ (105), $7.5 \%(9)$ and $5.0 \%$ (6), respectively. Nonidentification did not occur. Of note, the analysis of 6 S. epidermidis isolates $(6 / 53,11.3 \%)$ resulted in low-level discriminations - multiple identifications as $S$. hominis, S. epidermidis, or Aerococcus viridans with low percent probabilities, suggesting $S$. hominis with the highest percent probability followed by S. epidermidis (M123, M124, M125, M126, M127, M137).

\section{Identification using Crystal GP}

Crystal GP is not as fully automated as the other two, as the reading of the biochemical reaction depends on manual reading. In order to prevent the bias resulting from manual reading, the biochemical reaction results were read by two different technicians, followed by repeated tests on wrongly identified isolates or those with multiple identifications or nonidentifications. The identification results of 81 isolates $(67.5 \%)$ using the Crystal GP system agreed with those of the MicroSeq 500 system with confidence factors of more than 0.9 (correct identifications). In total, $23.3 \%$ (28) were misidentified, and 9.2\% (11) could not be identified (nonidentification). Low-level discrimination was not observed. Misidentifications at the genus level occurred for $1 S$. epidermidis and $1 S$. hominis isolate (M112, M216). The Crystal GP system correctly identified all the isolates of $S$. cohnii, $S$. haemolyticus, $S$. saprophyticus, S. simulans, and S. warneri.

\section{Discussion}

With the increased attention being given to the clinical significance of CNS, clinical laboratories must correctly identify CNS at the species level by using reliable and reproducible methods. Because clinical microbiology lab- 
Table I: Clinical isolates and identification results from MicroScan, VITEK2, and Crystal GP

\begin{tabular}{|c|c|c|c|c|c|}
\hline Clinical Isolates (using MicroSeq 500) & No of isolates & Correct Identification ${ }^{a}$ & Low-level Discrimination ${ }^{b}$ & Misidentificationc & Nonidentification \\
\hline & & \multicolumn{4}{|c|}{ MicroScan } \\
\hline S. capitis & 16 & $13(81.3 \%)$ & - & $3(18.8 \%)$ & - \\
\hline S. caprae & 4 & - & - & $4(100.0 \%)$ & - \\
\hline S. cohnii & 3 & I (33.3\%) & I (33.3\%) & I (33.3\%) & - \\
\hline S. epidermidis & 53 & $51(96.2 \%)$ & - & $2(3.8 \%)$ & - \\
\hline S. haemolyticus & 4 & $3(75.0 \%)$ & I (25.0\%) & - & - \\
\hline S. hominis & 25 & $23(92.0 \%)$ & $2(8.0 \%)$ & - & - \\
\hline S. lugdunensis & 6 & $2(33.3 \%)$ & I (16.7\%) & $3(50.0 \%)$ & - \\
\hline S. saprophyticus & I & I (I00.0\%) & - & - & - \\
\hline S. simulans & 2 & $2(100.0 \%)$ & - & - & - \\
\hline S. warneri & 6 & $3(50.0 \%)$ & $3(50.0 \%)$ & - & - \\
\hline \multirow[t]{2}{*}{ Subtotal } & 120 & 99 (82.5\%) & $8(6.7 \%)$ & $13(10.8 \%)$ & - \\
\hline & & \multicolumn{4}{|c|}{ VITEK 2} \\
\hline S. capitis & 16 & $16(100.0 \%)$ & - & - & - \\
\hline S. caprae & 4 & $3(75.0 \%)$ & - & I (25.0\%) & - \\
\hline S. cohnii & 3 & $3(100.0 \%)$ & - & - & - \\
\hline S. epidermidis & 53 & 47 (88.7\%) & $6(11.3 \%)$ & - & - \\
\hline S. haemolyticus & 4 & $4(100.0 \%)$ & - & - & - \\
\hline S. hominis & 25 & $22(88.0 \%)$ & I (4.0\%) & $2(8.0 \%)$ & - \\
\hline S. lugdunensis & 6 & 5 (83.3\%) & I (16.7\%) & - & - \\
\hline S. saprophyticus & I & I (100.0\%) & - & - & - \\
\hline S. simulans & 2 & I (50.0\%) & I (50.0\%) & - & - \\
\hline S. warneri & 6 & $3(50.0 \%)$ & - & $3(50.0 \%)$ & - \\
\hline \multirow[t]{2}{*}{ Subtotal } & 120 & $105(87.5 \%)$ & 9 (7.5\%) & $6(5.0 \%)$ & - \\
\hline & & \multicolumn{4}{|c|}{ Crystal GP } \\
\hline S. capitis & 16 & $13(81.3 \%)$ & - & $3(18.8 \%)$ & - \\
\hline S. caprae & 4 & - & - & $4(100.0 \%)$ & - \\
\hline S. cohnii & 3 & $3(100.0 \%)$ & - & - & - \\
\hline S. epidermidis & 53 & 49 (92.5\%) & - & $3(5.7 \%)$ & I (1.9\%) \\
\hline S. haemolyticus & 4 & $4(100.0 \%)$ & - & - & - \\
\hline S. hominis & 25 & I (4.0\%) & - & 15 (60.0\%) & $9(36.0 \%)$ \\
\hline S. lugdunensis & 6 & $2(33.3 \%)$ & - & $3(50.0 \%)$ & I (I6.7\%) \\
\hline S. saprophyticus & 1 & I (100.0\%) & - & - & - \\
\hline S. simulans & 2 & $2(100.0 \%)$ & - & - & - \\
\hline S. warneri & 6 & $6(100.0 \%)$ & - & - & - \\
\hline Subtotal & 120 & $81(67.5 \%)$ & - & $28(23.3 \%)$ & II (9.2\%) \\
\hline
\end{tabular}

Correct identification ${ }^{a}$ : single, unambiguous, correct identification at the species level Low-level discrimination ${ }^{b}$ : two or more possible species level identification including the correct one Misidentificationc: genus or species-level identification different from that obtained with the reference method Nonidentification ${ }^{d}$ : no identification or unidentification

oratories are increasingly becoming dependent on automated systems, the accuracy of these systems must be investigated. In the present study, the accuracy of 3 phenotypic identification systems for CNS identification was tested and compared with the sequencing of 16S rRNA.

MicroScan and VITEK 2 showed similar concordance rate to MicroSeq 500. VITEK 2 showed a slightly higher "correct identification" rate ( $82.5 \%$ vs. $87.5 \%$, respectively). When the low-level discrimination results that included the correct identification were considered together, the concordance rates were $89.2 \%(107 / 120)$ for MicroScan and $95.0 \%(114 / 120)$ for VITEK 2. Crystal GP had the lowest correct identification rate (67.5\%). All 3 systems had misidentifications: single but incorrect identification results with higher than acceptable probability of over $85 \%$, which is consistent with the system manufacturer's reported homology value without additional verification, or with higher than a confidence factor of 0.9. This indicates that CNS identifications made using these pheno- 
Table 2: Identification results with percent probability or confidence factor of single wrong identification or multiple identifications in three phenotypic systems

\begin{tabular}{|c|c|c|c|c|}
\hline & MicroSeq 500 & MicroScan & VITEK2 & Cystal GP \\
\hline MI02 & S. epidermidis & S. epidermidis $91.36 \%$ & S. epidermidis $97.10 \%$ & S. intermedius \\
\hline MI04 & S. epidermidis & S. xylosus $95.12 \%$ & S. epidermidis $99.00 \%$ & S. epidermidis \\
\hline MI06 & S. epidermidis & S. aureus $87.13 \%$ & S. epidermidis $99.00 \%$ & S. epidermidis \\
\hline MI07 & S. capitis & S. simulans $88.95 \%$ & S. capitis $98.78 \%$ & S. capitis \\
\hline MII2 & S. epidermidis & S. epidermidis $99.99 \%$ & S. epidermidis $99.00 \%$ & K. sedentarius \\
\hline MII7 & S. lugdunensis & S. haemolyticus $90.39 \%$ & S. lugdunensis $99.00 \%$ & Nonidentification \\
\hline MII8 & S. lugdunensis & S. haemolyticus $96.86 \%$ & S. lugdunensis $90.37 \%$ & S. haemolyticus \\
\hline MI20 & S. hominis & S. hominis $94.57 \%$ & S. hominis $95.00 \%$ & Nonidentification \\
\hline MI23 & S. epidermidis & S. epidermidis $98.20 \%$ & $\begin{array}{l}\text { S. hominis, S. epidermidis, A. viridans } \\
33.82 \%, 33.09 \%, 33.09 \%\end{array}$ & S. epidermidis \\
\hline MI24 & S. epidermidis & S. epidermidis $99.25 \%$ & S. hominis, S. epidermidis $50.27 \%, 49.73 \%$ & Nonidentification \\
\hline MI25 & S. epidermidis & S. epidermidis $98.20 \%$ & S. hominis, S. epidermidis $50.27 \%, 49.73 \%$ & S. epidermidis \\
\hline MI26 & S. epidermidis & S. epidermidis $99.99 \%$ & $\begin{array}{l}\text { S. hominis, S. epidermidis, A. viridans } \\
33.71 \%, 33.33 \%, 32.96 \%\end{array}$ & S. epidermidis \\
\hline MI27 & S. epidermidis & S. epidermidis $99.99 \%$ & S. hominis, S. epidermidis $50.28 \%, 47.92 \%$ & S. epidermidis \\
\hline$M 128$ & S. hominis & S. hominis $94.58 \%$ & S. hominis $94.86 \%$ & S. saprophyticus \\
\hline MI30 & S. capitis & S. epidermidis $90.20 \%$ & S. capitis $92.00 \%$ & S. capitis \\
\hline MI37 & S. epidermidis & S. epidermidis $95.28 \%$ & S. hominis, S. epidermidis $50.56 \%, 49.44 \%$ & S. epidermidis \\
\hline MI38 & S. capitis & S. capitis $95.28 \%$ & S. capitis $98.86 \%$ & S. auricularis \\
\hline MI39 & S. capitis & S. capitis $99.60 \%$ & S. capitis $98.86 \%$ & S. hominis \\
\hline MI4I & S. epidermidis & S. epidermidis $99.99 \%$ & S. epidermidis $98.16 \%$ & S. hominis \\
\hline MI49 & S. capitis & S. epidermidis $90.20 \%$ & S. capitis $92.00 \%$ & S. capitis \\
\hline MI53 & S. hominis & S. hominis $97.44 \%$ & S. hominis $99.00 \%$ & Nonidentification \\
\hline MI54 & S. hominis & S. hominis $94.62 \%$ & S. hominis $98.35 \%$ & Nonidentification \\
\hline MI55 & S. hominis & S. hominis $97.44 \%$ & S. hominis $97.21 \%$ & $\begin{array}{l}\text { S. warneri, S. saprophyticus } 0.5574 \text {, } \\
0.4415\end{array}$ \\
\hline MI56 & S. hominis & S. hominis $95.00 \%$ & S. hominis $97.00 \%$ & S. saprophyticus \\
\hline MI57 & S. warneri & $\begin{array}{l}\text { S. warneri, S. cohnii, S. capitis, etc. } 60.92 \% \text {, } \\
\text { 15.78\%, } 12.67 \% \text {. }\end{array}$ & S. cohnii, S. vitulinus $50.54 \%, 49.46 \%$ & S. warneri \\
\hline MI58 & S. warneri & S. warneri $90.92 \%$ & S. cohnii $90.54 \%$ & S. warneri \\
\hline MI60 & S. hominis & $\begin{array}{l}\text { S. hominis, S. capitis, S. warneri, etc. } 37.58 \% \text {, } \\
24.33 \%, 20.21 \% \text {. }\end{array}$ & S. hominis $93.79 \%$ & Nonidentification \\
\hline MI6I & S. hominis & S. hominis $94.57 \%$ & S. hominis $96.98 \%$ & S. saprophyticus \\
\hline MI62 & S. caprae & S. hominis $92.35 \%$ & S. auricularis $90.40 \%$ & S. saprophyticus \\
\hline MI63 & S. hominis & S. hominis $98.82 \%$ & S. hominis $98.50 \%$ & Nonidentification \\
\hline MI66 & S. hominis & S. hominis $85.00 \%$ & S. hominis $98.82 \%$ & Nonidentification \\
\hline MI67 & S. hominis & S. hominis $93.05 \%$ & S. caprae $96.97 \%$ & S. caprae \\
\hline MI76 & S. simulans & S. simulans $96.77 \%$ & S. simulans, S. warneri $50.28 \%, 49.72 \%$ & S. simulans \\
\hline MI7 & S. hominis & S. simulans $99.09 \%$ & S. haemolyticus $96.74 \%$ & S. haemolyticus \\
\hline MI79 & S. hominis & S. epidermidis, S. hominis $68.34 \%, 31.66 \%$ & S. hominis $94.88 \%$ & Nonidentification \\
\hline MI80 & S. hominis & S. hominis $92.77 \%$ & S. hominis $93.56 \%$ & S. warneri \\
\hline MI8I & S. hominis & S. hominis $92.77 \%$ & S. hominis, S. warneri $50.27 \%, 49.73 \%$ & S. warneri \\
\hline MI82 & S. hominis & S. hominis $94.57 \%$ & S. hominis $95.00 \%$ & Nonidentification \\
\hline MI83 & S. hominis & S. hominis $88.68 \%$ & S. hominis $91.00 \%$ & S. saprophyticus \\
\hline MI84 & S. hominis & S. hominis $92.13 \%$ & S. hominis $99.00 \%$ & S. intermedius \\
\hline MI85 & S. hominis & S. hominis $96.01 \%$ & S. hominis $96.81 \%$ & S. capitis \\
\hline MI86 & S. hominis & S. hominis $98.50 \%$ & S. hominis $95.00 \%$ & S. aureus, S. intermedius \\
\hline M187 & S. hominis & S. hominis $98.50 \%$ & S. hominis $99.00 \%$ & Nonidentification \\
\hline M203 & S. hominis & S. hominis $97.44 \%$ & S. hominis $97.27 \%$ & S. cohnii \\
\hline M205 & S. warneri & $\begin{array}{l}\text { S. warneri, S. capitis, S. hominis } 86.37 \% \text {, } \\
7.96 \%, 5.67 \%\end{array}$ & S. saphrophyticus $98.03 \%$ & S. warneri \\
\hline M207 & S. lugdunensis & S. haemolyticus $98.08 \%$ & S. lugdunensis $99.00 \%$ & S. lugdunensis \\
\hline M208 & S. haemolyticus & $\begin{array}{l}\text { S. haemolyticus, S. simulans, S. warneri } \\
54.38 \%, 41.97 \%, 3.66 \%\end{array}$ & S. haemolyticus $94.74 \%$ & S. haemolyticus \\
\hline M209 & S. hominis & S. hominis $97.44 \%$ & S. hominis $97.76 \%$ & S. cohnii \\
\hline M210 & S. cohnii & S. cohnii, S. xylosus $67.28 \%, 29.12 \%$ & S. cohnii $99.00 \%$ & S. cohnii \\
\hline M2II & S. caprae & S. aureus, S. capitis $69.87 \%, 29.25 \%$ & S. caprae $98.60 \%$ & S. intermidius \\
\hline$M 212$ & S. capitis & S. capitis $99.11 \%$ & S. capitis $98.56 \%$ & S. vitulus \\
\hline$M 213$ & S. warneri & S. auricularis, S. hominis, S. warneri $45.13 \%$, & S. warneri $95.00 \%$ & S. warneri \\
\hline
\end{tabular}


Table 2: Identification results with percent probability or confidence factor of single wrong identification or multiple identifications in three phenotypic systems (Continued)

\begin{tabular}{lllll}
\hline M214 & S. lugdunensis & S. lugdunensis $98.81 \%$ & S. lugdunensis $95.00 \%$ & S. simulans \\
M215 & S. caprae & S. capitis, S. aureus $62.82 \%, 35.76 \%$ & S. caprae $99.00 \%$ & S. cohnii \\
M216 & S. hominis & S. hominis $97.44 \%$ & S. hominis $97.76 \%$ & M. kristinae \\
M22I & S. lugdunensis & S. lugdunensis, S. haemoiyticus, S. hominis & S. lugdunensis, S. warneri $50.00 \%, 50.00 \%$ & S. lugdunensis \\
& & $62.62 \%, 28.34 \%, 9.04 \%$ & & \\
M222 & S. lugdunensis & S. lugdunensis $96.30 \%$ & S. lugdunensis $91.26 \%$ & S. simulans \\
M224 & S. cohnii & S. xylosus $91.70 \%$ & S. cohnii $99.00 \%$ & S. cohnii \\
M225 & S. caprae & S. warneri, S. simulans, S. epidermidis $70.19 \%$, & S. caprae $99.00 \%$ & S. capitis
\end{tabular}

typic systems cannot always be considered accurate, regardless of the reported probability.

In MicroScan analysis, none of the $S$. caprae identifications were correct ( 3 low-level identifications and 1 misidentification). This is because the species $S$. caprae is not included in the MicroScan database, which illustrates the inappropriateness of this database. Ambiguous identifications, including low-level discriminations and misidentifications, were observed for 8 out of 10 species (with the exception of $S$. saprophyticus and $S$. warneri) with the MicroScan system, compared with 6 ambiguous identifications with the VITEK2 system and 5 with the Crystal GP system. This finding was consistent with results reported by other researchers who found that the MicroScan system has greater accuracy in the identification of S. epidermidis and $S$. saprophyticus than for identification of other species [20-22]. The other notable finding of the present study is that $1 \mathrm{~S}$. epidermidis isolate from blood culture was misidentified as $S$. aureus with $87.5 \%$ probability, which could have delayed appropriate treatment for the patient.

In VITEK 2 analysis, low-level discriminations of 6 S. epidermidis isolates (11.3\% of $S$. epidermidis isolates) occurred, compared to higher correct identification rates with the MicroScan and Crystal GP systems (96.2\% and $92.5 \%$, respectively). Some researchers reported different sort of difficulties in identifying $S$. epidermidis with VITEK $2[9,23,24]$. However, others reported high accuracy of VITEK 2 for the identification of staphylocccal species $[25,26]$. The performance of VITEK 2 in the identification of CNS including $S$. epidermidis seems not to be determined yet, and the additional testing such as manual biochemical testing or sequencing is required for the accurate diagnosis.

The Crystal GP system provides no guidelines for the interpretation of the confidence factor, therefore, all isolates with confidence factors lower than 0.9 were retested. In spite of repeated testing, this system had the lowest correct identification rate and the highest rate of nonidentification.
One limitation of this study is that additional biochemical testing was not preformed. $16 \mathrm{~S}$ rRNA sequencing might not be a perfect method for interspecies discrimination. However, not all the suggested biochemical tests are available in many automated laboratories or based on our experience, manual testing could not resolve the ambiguity of CNS identification in many cases. To improve reliability in this study, all isolates with discrepancies or uncertain results were retested using isolates preserved in skim milk at $-70^{\circ} \mathrm{C}$.

\section{Conclusion}

None of the tested systems (MicroScan, VITEK 2, Crystal GP) are able to accurately identify all of the staphylococcal species evaluated in this study. Additional verification via molecular testing or suggested biochemical tests may prove helpful for correct CNS identification.

\section{Methods \\ Clinical isolates}

A total of 120 clinical isolates ( 95 blood culture isolates, 25 skin isolates from pitted keratolysis patients) were used in this study [27]. To exclude simple contaminants, only isolates of blood cultures that grew in more than 2 of 3 blood cultures were included. The pitted keratolysis specimen was obtained after repeated washing of the lesion with normal saline by scratching the lesion with a sterile blade into a sterile conical tube. The isolates were tested within 24 hours. For additional tests or repeated tests, the isolates were suspended in skim milk, stored at $-70^{\circ} \mathrm{C}$ and retested. All of the isolates were confirmed to be CNS by use of 16 S rRNA sequencing with MicroSeq 500 software (version 2.0) with over $97 \%$ matches.

\section{Phenotypic identification of CNS}

Three phenotypic systems were evaluated: MicroScan Pos Combo panel type 1A (Dade Behring, West Sacramento, CA, USA), VITEK 2 GP-ID cards (bioMérieux, Marcy l'Etoile, France), and Crystal Gram Positive ID systems (Becton Dickinson, Sparks, MD, USA). Bacterial suspensions were prepared from well-isolated colonies. The colonies were suspended in the broth provided by each company, and the turbidity was adjusted to $0.5 \mathrm{McFar}-$ land. MicroScan Pos Combo panel type 1A was inocu- 
lated according to the manufacturer's recommendations and processed with a MicroScan Walkaway 96 apparatus. The blood culture isolates were analyzed using a MicroScan LabPro system (version 2.0) and the skin isolates were analyzed with version 2.01. VITEK 2 GP-ID cards were used in combination with a VITEK 2 system (version 4.02) for all isolates. The Crystal GP results were read manually by 2 different technicians using the Crystal GP Identification Color Chart.

The MicroScan system classifies identification results into 4 categories by percent probability: species identified with high probability $(>85 \%)$, species identified with low probability $(<85 \%)$, very rare biotype, and group ID ( $>$ $85 \%$ but genus or group identified only). The VITEK 2 system categorizes the identification results by probability: excellent (96\%-99\%), very good (93\%-95\%), good (89\%-92\%), acceptable (85\%-88\%), low discrimination (2-3 identifications with $100 \%$ probability in total), and nonidentification. The Crystal GP system gives confidence factors instead of probability; however, there are no such criteria in terms of the confidence factor. When the confidence factor is low, Crystal GP reports non-identification, which necessitates additional manual testing.

The results of incorrect identifications (not concordant to that of MicroSeq 500) regardless of probability, identification with unacceptable probability (lower than $85 \%$ for MicroScan and VITEK 2) or low confidence factor (lower than 0.9 for Crystal GP), or nonidentification were retested with colonies that were stored in skim milk at $70^{\circ} \mathrm{C}$. Those with incorrect, or consistently unacceptable or nonidentifiable results are listed in Table 2. The confidence factor of the Crystal GP system is not included, as there are no guidelines for these interpretations.

\section{I6S rRNA sequencing}

To extract bacterial DNA, InstaGene Matrix (Bio-Rad Laboratories, Hercules, CA, USA) was used. Two or 3 colonies of each isolate were suspended in $100 \mu \mathrm{l}$ sterile distilled water and centrifuged for $5 \mathrm{~min}$ at 15,000 rpm. A $200 \mu \mathrm{l}$ aliquot of InstaGene Matrix (Bio-Rad Laboratories, Hercules, CA, USA) was added to the sediment. The mixture was heat-lysed for $5 \mathrm{~min}$ at $100^{\circ} \mathrm{C}$, cooled at room temperature, and centrifuged for $3 \mathrm{~min}$ at 15,000 rpm. The supernatant $(2.5 \mu \mathrm{l})$ of each bacterial extract was used for successive amplification procedures. Polymerase chain reaction (PCR) was conducted in a total volume of $25 \mu \mathrm{l}$ containing $2.5 \mathrm{mM}$ dNTP, 10 pmol of each PCR primer, 0.6 units Taq polymerase, $2.5 \mu \mathrm{l} 10 \times$ PCR buffer with 15 $\mathrm{mM} \mathrm{MgCl} 2$ (Takara Bio, Inc., Shiga, Japan), and $2.5 \mu \mathrm{l}$ template. In-house primers were designed using the LightCycler Probe Design software (version 2.0) (Roche, Penzberg, Germany), using published studies as a reference $[8,9]$. The primers used for amplification were MSQ-F (5'-
TGA AGA GTT TGA TCA TGG CTC AG-3') and MSQ-R (5'ACC GCG GCT GCT GGC AC-3'). The PCR conditions were as follows: $10 \mathrm{~min}$ of initial denaturation at $95^{\circ} \mathrm{C}$, followed by 35 cycles of annealing of $30 \mathrm{~s}$ at $95^{\circ} \mathrm{C}$, 30s at $60^{\circ} \mathrm{C}, 45 \mathrm{~s}$ at $72^{\circ} \mathrm{C}$, and a final 10 -minute extension at $72^{\circ} \mathrm{C}$. Gel electrophoresis was used to detect positive PCR signals and to confirm the length of the amplicon. Prior to sequencing, the PCR products were purified using ExoSAP-IT reagent (USB Corporation, Cleveland, Ohio, USA) according to the manufacturer's instructions. Forward and reverse sequencing reactions were conducted for each of the amplified products. The sequencing reaction consisted of $10 \mu \mathrm{l}$ MicroSeq 500 sequencing mix (containing $1.6 \mathrm{pmol}$ of MSQ-F and MSQ-R primers), $2.9 \mu \mathrm{l}$ sterile distilled water, and $1 \mu \mathrm{l}$ purified PCR product. Sequencing reactions were performed using Big Dye terminator reagents on an ABI Prism 3130xl Genetic Analyzer (Applied Biosystems Inc., Foster City, CA, USA) according to standard automated sequencer protocols.

\section{Sequence analysis}

MicroSeq 500 version 2.0 software was used for sequence analysis. The analysis steps were as follows: (i) the forward and reverse sequences were assembled into one consensus sequence, (ii) the consensus sequence was edited to resolve discrepancies between the 2 strands by evaluating the electropherograms, and (iii) the consensus sequence was compared with sequences in the MicroSeq 500 database. The comparison, using the full alignment tool of the MicroSeq 500 software, generated a list of the closest matches with a distance score, which indicates the percentage difference between the unknown sequence and the database sequence. Only when the consensus sequence had more than a $97 \%$ match with that of the MicroSeq 500 results, the identification results were considered to be acceptable $[7,10]$.

\section{Analysis of the results}

The results of 16S rRNA sequencing and MicroSeq 500 analysis were regarded as the definitive identification [1012,16-19]. The species-level identification and percent probability from phenotypic systems were all taken into consideration. The concordance of the results of the phenotypic systems with those of MicroSeq 500 were classified into 4 categories according to the following definitions: (i) correct identification (unambiguous, single identification identical to that of MicroSeq 500 at the species level, with $>85 \%$ probability in MicroScan and VITEK 2 or 0.9 confidence factor); (ii) low-level discrimination (2 or more possible species-level identifications, including the correct one, with probability $<85 \%$ in MicroScan and VITEK 2 or the low confidence factor under 0.9 in Crystal GP); (iii) misidentification (genuslevel or species-level identification different from that obtained with the reference method) and (iv) nonidenti- 
fication (isolates that were unable to be identified at the species level in spite of the repeated testing).

\section{Authors' contributions}

MK designed the study, helped with the phenotypic identification and molecular studies, and wrote the manuscript. SHC performed sample collection and phenotypic identification. SRH, HK and JSP carried out the molecular genetic studies, participated in the sequence alignment. MWS, DHL, JS and ECK helped design the study and helped with technical issues. KUP conceived of study, and participated in its design and coordination, helped to draft the manuscript and reviewed the manuscript. All of the authors have read and approved the final manuscript.

\section{References}

I. von Eiff C, Peters G, Heilmann C: Pathogenesis of infections due to coagulase-negative staphylococci. Lancet Infectious Dis 2000, 2:677-685.

2. Longauerova $A$ : Coagulase negative staphylococci and their participation in pathogenesis of human infections. Bratisl Lek Listy 2006, 107:448-452.

3. Pfaller MA, Herwaldt LA: Laboratory, clinical, and epidemiological aspects of coagulase-negative staphylococci. Clin Microbiol Rev 1998, I(3):28I-299.

4. Heikens E, Fleer A, Paauw A, Florijn A, Fluit AC: Comparison of genotypic and phenotypic methods for species-level identification of clinical isolates of coagulase-negative staphylococci. J Clin Microbiol 2005, 43:2286-2290.

5. Weinstein MP, Mirrett S, Van Pelt L, McKinnon M, Zimmer BL, Kloos $W$, Reller LB: Clinical importance of identifying coagulasenegative staphylococci isolated from blood cultures: Evaluation of MicroScan Rapid and Dried Overnight Gram-Positive Panels versus a conventional reference method. J Clin Microbiol 1998, 36:2089-2092.

6. Becker K, Harmsen D, Mellmann A, Meier C, Schumann P, Peters G, von Eiff $C$ : Development and evaluation of quality-controlled ribosomal sequence database for 165 ribosomal DNA-based identification of Staphylococcus species. J Clin Microbiol 2004, 42:4988-4995

7. Bosshard PP, Żbinden R, Abels S, Böddinghaus B, Altwegg M, Böttger EC: 165 rRNA gene sequencing versus the API 20 NE System and the VITEK 2 ID-GNB Card for identification of nonfermenting gram-negative bacteria in the clinical laboratory. J Clin Microbiol 2006, 44: I359-I 366

8. von Eiff $C$, Peters G, Becker K: The small colony variant (SCV) concept - the role of staphylococcal SCVs in persistent infections. Injury 2006, 37:S26-S33.

9. von Eiff C, Vaudaux P, Kahl BC, Lew D, Emler S, Schmidt A, Peters G, Proctor RA: Bloodstream infections caused by small-colony variants of coagulase-negative staphylococci following pacemaker implantation. Clin Infect Dis 1999, 29:932-4.

10. Clarridge JE 3rd: Impact of I6S rRNA gene sequence analysis for identification of bacteria on clinical microbiology and infectious disease. Clin Microbiol Rev 2004, 17:840-862.

II. Woo PC, Ng KH, Lau SK, Yip KT, Fung AM, Leung KW, Tam DM, Que TL, Yuen KY: Usefulness of MicroSeq 500 I 65 ribosomal DNA-based bacterial identification system for identification of clinically significant bacterial isolates with ambiguous biochemical profiles. J Clin Microbiol 2003, 4I:1996-200 I.

12. Fontana C, Favaro M, Pelliccioni M, Pistoia ES, Favalli C: Use of the MicroSeq 500 I 6 S rRNA gene-based sequencing for Identification of bacterial isolates that commercial automated systems failed to identify correctly. J Clin Microbiol 2005, 43:615-619.

13. Carretto E, Barbarini D, Couto I, De Vitis D, Marone P, VerhoefJ, De Lencastre $H$, Brisse S: Identification of coagulase-negative staphylococci other than Staphylococcus epidermidis by automated ribotyping. Clin Microbiol Infect 2005, I I:I77-I84.
14. Layer F, Ghebremedhin B, Moder KA, König W, König B: Comparative study using various methods for identification of Staphylococcus species in clinical specimens. J Clin Microbiol 2006, 44:2824-2830.

15. Aldea-Mansilla C, García de Viedma D, Cercenado E, Martín-Rabadán $P$, Marín M, Bouza E: Comparison of phenotypic with genotypic procedures for confirmation of coagulase-negative staphylococcus catheter-related bloodstream infections. J Clin Microbiol 2006, 44:3529-3532.

16. Hall L, Doerr KA, Wohlfiel SL, Roberts GD: Evaluation of the MicroSeq System for identification of Mycobacteria by 165 ribosomal DNA sequencing and its integration into a routine clinical Mycobacteriology laboratory. J Clin Microbiol 2003, $41:$ : $447-1453$.

17. Patel JB, Leonard DG, Pan X, Musser JM, Berman RE, Nachamkin I: Sequence-based identification of Mycobacterium species using the MicroSeq 500 I6S rDNA Bacterial Identification System. J Clin Microbiol 2000, 38:246-25I.

18. Tang YW, Ellis NM, Hopkins MK, Smith DH, Dodge DE, Persing DH: Comparison of phenotypic and genotypic techniques for identification of unusual aerobic pathogenic gram-negative bacilli. J Clin Microbiol 1998, 36:3674-3679.

19. Cloud IL, Conville PS, Croft A, Harmsen D, Witebsky FG, Carroll KC: Evaluation of Partial I6S ribosomal DNA sequencing for identification of Nocardia species by using the MicroSeq $\mathbf{5 0 0}$ System with an expanded database. J Clin Microbiol 2004, 42:578-584

20. Hussain Z, Stoakes L, Stevens DL, Schieven BC, Lannigan R, Jones C: Comparison of the MicroScan System with the API StaphIdent System for species identification of coagulase-negative staphylococci. / Clin Microbiol 1986, 23: I 26- 128.

2I. Skulnick M, Patel MP, Low DE: Evaluation of five commercial systems for identification of coagulase-negative staphylococci to species level. Eur / Clin Microbiol Infect Dis 1989, 8:1001-I003.

22. Stoakes L, Schieven BC, Ofori E, Ewan P, Lannigan R, Hussain Z: Evaluation of MicroScan Rapid Pos Combo Panels for identification of staphylococci. J Clin Microbiol 1992, 30:93-95.

23. Ben-Ami R, Navon-Venezia S, Schwartz D, Schlezinger $Y$, Mekuzas $Y$, Carmeli $Y$ : Erroneous reporting of coagulase-negative staphylococci as Kocuria spp. by Vitek 2 System. J Clin Microbiol 2005, 43: $1448-1450$.

24. Ligozzi M, Bernini C, Bonora MG, De Fatima M, Zuliani J, Fontana R Evaluation of the VITEK 2 System for identification and antimicrobial susceptibility testing of medically relevant grampositive cocci. J Clin Microbiol 2002, 40: 168I-1686.

25. Eigner U, Schmid A, Wild U, Bertsch D, Fahr A-M: Analysis of the Comparative Workflow and Performance Characteristics of the VITEK 2 and Phoenix Systems. J Clin Microbiol 2005, 43:3829-3834.

26. Delmas J, Chacornac JP, Robin F, Giammarinaro P, Talon R, Bonnet R: Evaluation of the Vitek 2 System with a Variety of Staphylococcus Species. J Clin Microbiol 2008, 46:3 I I-3I3.

27. Kim BJ, Park KY, Kim JY, Ahn JY, Won CH, Lee JH, Rho NH, Kim SH, Cho SY, Kwon OS, Huh CH, Youn SW, Kim MN, Ro BY: Comparative study of benzoyl peroxide versus clindamycin phosphate in treatment of pitted keratolysis. Korean J Med Mycol 2005, 10:144-150.

Publish with Bio Med Central and every scientist can read your work free of charge

"BioMed Central will be the most significant development for disseminating the results of biomedical research in our lifetime."

Sir Paul Nurse, Cancer Research UK

Your research papers will be:

- available free of charge to the entire biomedical community

- peer reviewed and published immediately upon acceptance

- cited in PubMed and archived on PubMed Central

- yours - you keep the copyright 